This article was downloaded by: [EBSCOHost EJS Content Distribution]

On: 15 April 2009

Access details: Access Details: [subscription number 902156990]

Publisher Informa Healthcare

Informa Ltd Registered in England and Wales Registered Number: 1072954 Registered office: Mortimer House, 37-41 Mortimer Street, London W1T 3JH, UK

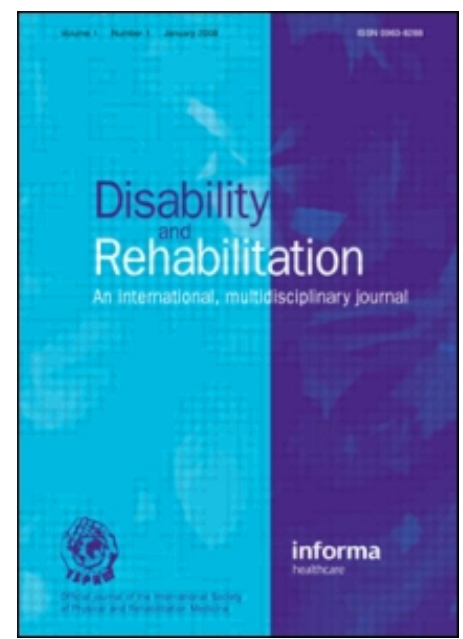

Disability \& Rehabilitation

Publication details, including instructions for authors and subscription information:

http://www.informaworld.com/smpp/title content=t713723807

\title{
Prediction of hand function after occupational hand injury by evaluation of initial anatomical severity
}

Chia-Ling Lee ab; Ming-Yi Wu c; Jer-Hao Chang c; Haw-Yen Chiu de; Ching-Hsiang Chiang d; Mao-Hsiung Huang af; Yue-Liang Guo bg

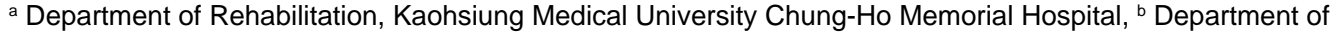
Environmental and Occupational Medicine, ${ }^{\circ}$ Department of Occupational Therapy, National Cheng-Kung University, Tainan ${ }^{d}$ Division of Plastic Surgery, National Cheng-Kung University Hospital, Tainan ${ }^{e}$ Division of Plastic Surgery, Chiali General Hospital, Tainan ${ }^{+}$Department of Rehabilitation, Faculty of Medicine, College of Medicine, Kaohsiung Medical University, Kaohsiung ${ }^{9}$ Department of Environmental and Occupational Medicine, National Taiwan University, Taipei, Taiwan

First Published:2008

To cite this Article Lee, Chia-Ling, Wu, Ming-Yi, Chang, Jer-Hao, Chiu, Haw-Yen, Chiang, Ching-Hsiang, Huang, Mao-Hsiung and Guo, Yue-Liang(2008)'Prediction of hand function after occupational hand injury by evaluation of initial anatomical severity',Disability \& Rehabilitation, $30: 11,848-854$

To link to this Article: DOI: $10.1080 / 09638280701419375$

URL: http://dx.doi.org/10.1080/09638280701419375

\section{PLEASE SCROLL DOWN FOR ARTICLE}

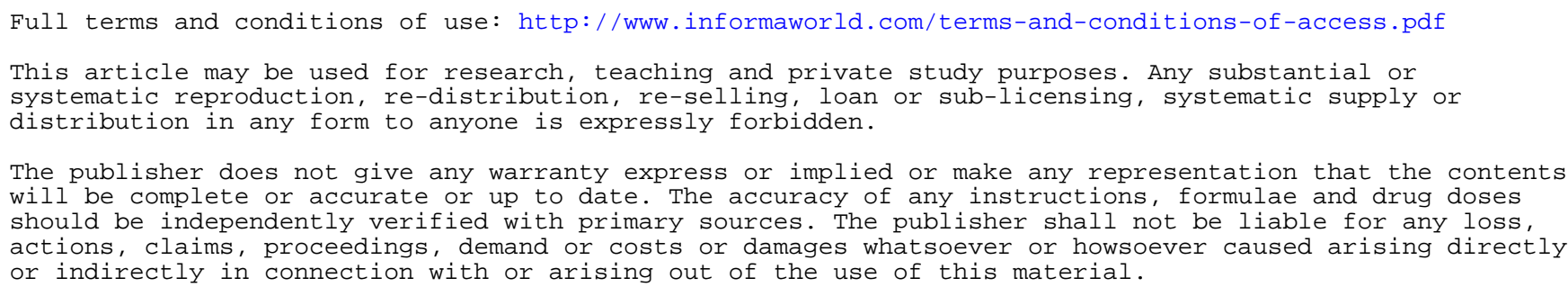




\title{
Prediction of hand function after occupational hand injury by evaluation of initial anatomical severity
}

\author{
CHIA-LING LEE ${ }^{1,3}$, MING-YI WU ${ }^{4}$, JER-HAO CHANG ${ }^{4}$, HAW-YEN CHIU ${ }^{5,6}$, \\ CHING-HSIANG CHIANG ${ }^{5}$, MAO-HSIUNG HUANG ${ }^{1,2} \&$ YUE-LIANG GUO ${ }^{3,7}$
}

${ }^{1}$ Department of Rehabilitation, Kaohsiung Medical University Chung-Ho Memorial Hospital, ${ }^{2}$ Department of Rehabilitation, Faculty of Medicine, College of Medicine, Kaohsiung Medical University, Kaohsiung, ${ }^{3}$ Department of Environmental and Occupational Medicine, ${ }^{4}$ Department of Occupational Therapy, National Cheng-Kung University, Tainan, ${ }^{5}$ Division of Plastic Surgery, National Cheng-Kung University Hospital, Tainan, ${ }^{6}$ Division of Plastic Surgery, Chiali General Hospital, Tainan, and ${ }^{7}$ Department of Environmental and Occupational Medicine, National Taiwan University, Taipei, Taiwan

Accepted April 2006

\begin{abstract}
Purpose. To examine the relationship between initial anatomic severity evaluated by the Hand Injury Severity Scoring (HISS) system and recovered hand function evaluated by the Purdue Pegboard after occupational hand injury.

Method. In the retrospective cohort study, 95 patients hospitalized between 1 January 2000 and 31 December 2003 for surgery due to occupational hand injury were recruited. The HISS scores were obtained by chart review by a surgeon. The Purdue Pegboard was performed at least 6 months after the injury by well-trained occupational therapists. The Purdue Pegboard scores were compared with the initial HISS scores by simple regression, multiple regression and logistic regression analysis.

Results. The total HISS score was negatively correlated with scores of the Purdue Pegboard subtests, including injuredhand, both-hands, and assembly. The risk of having low injured-hand score $(<13)$ was significantly increased in workers with initial major severity (HISS $\geq 101$ ), with OR 9.57 (95\% CI 1.4-94.8). The risk of having low both-hands score ( $<10$ ) was significantly increased in workers with initial severe and major severity (HISS of $51-100$ and $\geq 101$ ), with OR 4.5 (95\% CI $1.1-21.8)$ and OR 25 (95\% CI 3.5-263). The risk of having low assembly score $(<25)$ was significantly increased in workers with initial major severity (HISS $\geq 101$ ), with OR 9.0 (95\% CI $1.3-72.5$ ).

Conclusion. The study showed that after occupational hand injury, initial anatomic severity evaluated by the HISS system could predict hand function after recovery.
\end{abstract}

Keywords: Hand injury, hand function, HISS, the Purdue Pegboard

\section{Introduction}

Work-related injury creates cost not only in medical burden but also in loss of productivity $[1,2]$. In Taiwan, the incidence of work-related injuries was as high as 3.6-5.9 persons per thousand persons per year between 1991 and 1999, and 6.1-6.6 between 2000 and 2003 [3]. Furthermore, 'clamped and rolled' constituted the second frequent type of occupational injury, following traffic accident, and caused the most disability as the result of occupational injury in 2003. The most frequent body part of occupational injury was finger and hand [3]. A previous study revealed the economic impact of hand injuries, especially labor costs [4]. Therefore, occupational hand injury is an important issue in Taiwan.

The prediction of recovery after injury is important to clinical practitioners including surgeons, rehabilitation staff and occupational practitioners. A thorough evaluation of the recovery after hand injury would offer a workable treatment program [5]. Surgeons have to decide what surgical approach to use; rehabilitative staff have to set the goal and make the strategy of training. Occupational practitioners have to evaluate the work ability and the possibility of return-to-work. Thus, predicting the recovery after

Correspondence: Yue-Liang Guo, MD, PhD, Department of Environmental and Occupational Medicine, National Taiwan University, 1 Sec 1, Jen-ai Road, Taipei 100, Taiwan. Tel: 8862 33228215. Fax: 8862 33228216. E-mail: leonguo@ha.mc.ntu.edu.tw 
occupational hand injury provides important information for the care of workers with occupational hand injuries.

The hand is a complicated structure. Its performance depends on its sensory system and motor system, including skeletal system and neuromuscular system. Many studies had tried to reveal the effect of individual structures or factors [6-10]. Campbell and Kay tried to include all the possible involving anatomic components and get comprehensive evaluation. The Hand Injury Severity Scoring (HISS) system was developed in 1996. This system evaluated the four systems, including integument (skin and nail), skeletal (bones, joints and ligaments), motor (tendons and intrinsic muscles) and neural (median, ulnar and digital nerves) components. They also used the HISS as a guide to predict outcome, such as return-to-work [11].

A previous study revealed significant correlation of the HISS score with the time interval between injury and the end of healing [12]. They also showed statistically significant correlation between the HISS score and the American Medical Association (AMA) impairment rating which was evaluated by loss of active movement [13]. Regarding correlation with disabilities evaluated by the Disabilities of the Arm, Shoulder and Hand (DASH), one subjective outcome measurement instruments for upper extremity symptoms and function by questionnaire [14,15], the results in two studies were somewhat contradictory. Mink van der Molen found that there was no statistically significant correlation between the HISS and the DASH [13], but Saxena found that these two were significantly correlated [16]. The International Classification of Functioning, Disability and Health (ICF) system [17] has been widely used to describe health-related state in many studies $[18,19]$. According to the ICF system, these previous studies focused on domain of the 'body functions and structures' (i.e., wound and tissue healing, AMA impairment rating) and domain of 'participation' (i.e., return-towork, DASH). As far as we know, there has been no direct comparison between the HISS score and hand function, which refers to domain of 'activity'.

Dexterity, or hand function refers to the ability to manipulate objects with the hands. Accuracy and speed are the parameters of measurement [20]. Many hand function tests were developed since the twenty century, including the Purdue Pegboard [21], O'Connor Dexterity Tests [22], Jebsen Test of Hand Function [23], Minnesota Rate of Manipulation Test [24], Smith Hand Function Test (Smith) [25], Bennett Hand Tool Dexterity Test [26], Nine Hole Peg Test [27], TEMPA [28], and so on. The Purdue Pegboard was first developed in 1948 by Joseph Tiffin, $\mathrm{PhD}$, an Industrial Psychologist in Purdue University [21,29]. It was initially developed for employee selection [21], but was later used more extensively in other vocational fields [30], as well as for the evaluation of hand function in various ailments [31-33]. Test-retest reliability was studied with fair to good reliability [34-36], despite of practice effect reported [35,36]. In our study, we used the Purdue Pegboard as an indicator of hand function after hand injury.

The aim of this study is to examine the correlation between initial anatomic severity after occupational hand injury, evaluated by the HISS system, and hand function after an adequate period of recovery, evaluated by the Purdue Pegboard.

\section{Methods}

Subjects

Patients hospitalized between 1 January 2000 and 31 December 2003 for surgery due to occupational hand injury in one tertiary referral centre in southern Taiwan were recruited. These patients were at least 6 months after injury, allowing complete recovery from the injury. They were clinically stable and no longer needed a splinting device. We excluded patients with poor communication (e.g., foreign language speaking only), no work while injured, age younger than 15 or older than 65 , hand injury beyond distal to carpus, bilateral hand injury, and previous severe hand injury. Left-handed patients accounted for only around $1 \%$, and thus excluded from analysis for the purpose of homogeneity of the study population.

Phone interview was conducted between August 2003 and December 2004 for demographic information. Informed consent was obtained for participation of this study. For those agreed to participate, clinic appointment was made for hand function testing. Written consent was again obtained at this visit.

\section{The Purdue Pegboard}

There are two types of manual dexterity (hand function), including gross and fine dexterity. The former one emphasizes more global movement, and the latter one requires fine, interdigital movements. The Purdue Pegboard measures these two.

The Purdue Pegboard is a board with four cups at the top to contain pins, collars and washers, and two rows of holes in the middle from top to bottom. Five separate scores from four subtests were obtained in formal test, including right hand, left hand, both hands, right + left + both hands, and assembly [37]. The one-hand and both-hands subtests measure unilateral and bilateral gross manual dexterity, and the assembly subtest measures fine dexterity $[21,35]$. We replaced the right hand or left hand subtest with injured-hand subtest. Therefore, scores 
of injured-hand, both-hands and assembly subtests were used in our study for the evaluation of hand function after adequate recovery period after hand injury.

\section{The injured-hand subtest}

In this test, the participant was asked to pick up one pin at a time with their injured hand from the cup on the same side as their injured hand and then place each pin into a series of holes, running adjacent to the cup, from top to bottom. The score referred to the number of the pins he or she moved in 30 seconds.

\section{The both-hands subtest}

In this test, both hands were used at the same time. The participant was asked to pick up one pin with right hand from the right-hand cup and pick up one pin with left hand from the left-hand cup, at the same time, and then place the pins down the rows of holes. The score referred to the pairs of pins he or she moved in 30 seconds.

\section{The assembly subtest}

In this more complicated test, finger dexterity was tested. In step one, the participants picked up one pin from the dominant-hand cup with their dominant hand and placed the pin in the top hole in the dominant-hand row. In step two, they picked up a washer with their non-dominant hand and dropped the washer over the pin. In step three, they picked up a collar with their dominant hand and dropped the collar over the pin. In step four, they picked up another washer with their non-dominant hand and dropped it over the collar. After completing the four steps, they moved on to the next holes. The score referred to the number of pins, washers or collars he or she moved in 60 seconds.

The norm was provided in several populations $[21,35,37]$. We adopted the one in which male hourly production workers were studied [37]. The hourly production workers represented the population in whom good hand function was especially required. We defined poor hand function as getting scores lower than those of the 5 percentile of this population; those were 13 in the one hand (injuredhand) subtest, 10 in the both-hands subtest, and 25 in the assembly subtest.

\section{The Hand Injury Severity Scoring (HISS) system}

Medical records were reviewed by a hand surgeon. The chart records included photographs of the injury upon arrival at the emergency department and notes of the findings during operation. Initial emergency room evaluation and the operation records were translated into scores by using the HISS system [11]. The HISS system was developed by Campbell and Kay in 1996. Four components, integument, skeletal, motor and neural (ISMN), were evaluated over each ray. Weighting factor (according to the functional importance) was given to each ray. Besides, there were absolute values which required no modification in integument and neural components. There were also conditions in which the scores should be doubled. A score could be obtained for each component by summing up the weighted value of each ray and absolute value in each component. The total HISS score was thus obtained by summing up the scores of the four components.

According to original report, four categories of severity were also stated [11]. A HISS score of 20 or below was classified as 'Minor' severity, between 21 and 50 as 'Moderate' severity, between 51 and 100 as 'Severe' severity, and 101 and above as 'Major' severity.

\section{Statistics}

T-test or Chi-square test was used for examining the difference between two groups. T-test or simple regression was used for association between covariates and the hand function tests. Simple regression and multiple regression were used for correlation between the HISS scores and scores of the hand function tests. Logistic regression was used for evaluating the risk of poor hand function by initial severity. Potential covariates, including gender, age, and education, are known predictors for hand performance, and were included as adjusting covariates in the regression analyses. Whether injured hand was dominant could potentially affect the performance of hand, and was used as a covariate. In addition, uninjured-hand Purdue was included as an adjusting covariate, which served as an indicator of one's own hand ability. Odds ratio and 95\% confidence intervals of having poor hand function was calculated after adjusted for covariates.

\section{Results}

\section{Demographic data}

According to our inclusion criteria, 329 occupational hand injury patients were candidates in our study. Among them, 191 patients completed the phone interview, and 95 patients were able to visit our hospital for the hand function tests. There was no statistical difference in gender and age between patients with and without telephone interview. There was also no statistically significant difference in gender, age, marriage status and education between patients with and without the hand function tests. 
Among the 95 patients, 77 men and 18 women, who completed the study, all hand function studies were completed at least 6 months after the injury episode. The subjects had mean age of 39.4 years, and the most prevalent education level of senior high school (44.2\%). Forty two (44.2\%) suffered from dominant hand injury, and $53(55.8 \%)$ non-dominant hand injury (Table I). The most common injury type was 'clamped, crushed and rolled' (65.3\%).

\section{The HISS system}

The mean total HISS score was 47.3 (SD 36.0), median 37 (range 2-158). The mean scores of

Table I. Demographic characteristics and injury side in the study population.

\begin{tabular}{lccc}
\hline Variable & $n$ & $\%$ & Mean \pm SD \\
\hline Gender & & & \\
$\quad$ Female & 18 & 18.9 & \\
$\quad$ Male & 77 & 81.1 & \\
Age & & & $39.4 \pm 12.6$ \\
$\quad \leq 27$ & 23 & 24.2 & \\
$28-37$ & 23 & 24.2 & \\
$\quad 38-48$ & 24 & 25.3 & \\
$\quad 49$ & 25 & 26.3 & \\
Education & & & $11.1 \pm 3.5$ \\
$\quad$ Elementary school or less & 19 & 20.0 & \\
$\quad$ Junior high school & 15 & 15.8 & \\
$\quad$ Senior high school & 42 & 44.2 & \\
$\quad$ College & 19 & 20.0 & \\
Injured hand & & & \\
$\quad$ Dominant & 42 & 44.2 & \\
$\quad$ Non-dominant & 53 & 55.8 & \\
\hline
\end{tabular}

individual component and the case numbers (percentages) of involvement in each component are listed in Table II. Integument component was highly involved $(96.8 \%)$, but neural component was not $(28.4 \%)$. There was no significant association between the HISS scores and covariates (gender, age, education and dominant hand injury or not). The distribution of the HISS scores according to severity categories had reasonable representation, namely, most with moderate (HISS 21-50), and least with major severity (HISS $\geq 101$ ) (Table VI).

\section{The Purdue Pegboard}

Mean scores with standard deviation (SD) of injured-hand, both-hands and assembly subtests in the Purdue Pegboard are listed in Table III. Around one-third of patients got low scores in all the three subtests. The associations between covariates (gender, age, education, dominant or non-dominant hand injury) and hand function were evaluated. Age was negatively associated with assembly score, and education was positively associated with assembly score, but not other scores (Table IV).

\section{Correlation between scores of the HISS system and the Purdue Pegboard}

The total HISS score was significantly associated with scores of the injured-hand, both-hands and assembly subtests (see Figure 1). However, not all of the four components were associated with the subtests. After adjusting for covariates, scores of the three Purdue Pegboard subtests were still associated with the total

Table II. Distribution of scores of the HISS system among the 95 participants.

\begin{tabular}{|c|c|c|c|c|c|}
\hline Subtest & Mean \pm SD & Median & Range & Possible range & $\begin{array}{c}\text { Number } \\
\text { (percentage) of } \\
\text { involvement }^{\star}\end{array}$ \\
\hline Total & $47.3 \pm 36.0$ & 37 & $2-158$ & $0-1064$ & $95(100 \%)$ \\
\hline Integument & $19.7 \pm 15.4$ & 15 & $0-104$ & $0-472$ & $92(96.8 \%)$ \\
\hline Skeletal & $15.2 \pm 19.6$ & 8 & $0-116$ & $0-192$ & $71(74.7 \%)$ \\
\hline Motor & $8.6 \pm 13.9$ & 0 & $0-77$ & $0-256$ & $43(45.3 \%)$ \\
\hline Neural & $3.9 \pm 7.1$ & 0 & $0-24$ & $0-144$ & $27(28.4 \%)$ \\
\hline
\end{tabular}

^Involvement was defined as score of that component more than zero.

Table III. Distribution of scores of the Purdue Pegboard among the 95 participants as compared to normal workers.

\begin{tabular}{|c|c|c|c|c|c|}
\hline Subtest & Mean \pm SD & Median & Range & Possible Range & $\begin{array}{c}\text { Number } \\
\text { (percentage) of } \\
\text { low score }\end{array}$ \\
\hline Injured-hand & $12.7 \pm 3.3$ & 13 & $1-20$ & $0-25$ & $40(42 \%)$ \\
\hline Both-hands & $10.3 \pm 2.6$ & 11 & $2-15$ & $0-25$ & $34(36 \%)$ \\
\hline Assembly & $28.6 \pm 9.5$ & 28 & $4-52$ & $0-80$ & $34(36 \%)$ \\
\hline
\end{tabular}

${ }^{\star}$ Low score was defined as lower than 5 percentile among the norm from male hourly production workers [37]. 
Table IV. Association between covariates and the Purdue Pegboard scores.

\begin{tabular}{|c|c|c|c|c|c|c|c|}
\hline \multirow{2}{*}{ Covariate Purdue Pegboard } & \multirow[b]{2}{*}{$n$} & \multicolumn{2}{|c|}{ Injured-hand } & \multicolumn{2}{|c|}{ Both-hands } & \multicolumn{2}{|c|}{ Assembly } \\
\hline & & Mean & $p$ & Mean & $p$ & Mean & $p$ \\
\hline \multicolumn{8}{|l|}{ Gender } \\
\hline Female & 18 & 13.6 & 0.18 & 11.2 & 0.10 & 30.7 & 0.31 \\
\hline Male & 77 & 12.4 & & 10.1 & & 28.1 & \\
\hline \multicolumn{8}{|l|}{ Age } \\
\hline$\leq 27$ & 23 & 13.5 & 0.08 & 11.0 & 0.05 & 32.7 & $<0.01^{\star}$ \\
\hline $28-37$ & 23 & 13.0 & & 11.1 & & 31.9 & \\
\hline $38-48$ & 24 & 11.2 & & 9.7 & & 25.0 & \\
\hline$\geq 49$ & 25 & 13.0 & & 9.5 & & 25.2 & \\
\hline \multicolumn{8}{|l|}{ Education } \\
\hline Elementary school or less & 19 & 12.7 & 0.79 & 9.3 & 0.15 & 22.3 & $<0.01^{\star}$ \\
\hline Junior high school & 15 & 11.9 & & 9.8 & & 26.8 & \\
\hline Senior high school & 42 & 12.9 & & 10.8 & & 31.8 & \\
\hline College & 19 & 12.8 & & 10.7 & & 29.3 & \\
\hline \multicolumn{8}{|l|}{ Injured hand } \\
\hline Dominant & 42 & 12.9 & 0.62 & 10.4 & 0.68 & 27.9 & 0.51 \\
\hline Non-dominant & 53 & 12.5 & & 10.2 & & 29.2 & \\
\hline
\end{tabular}

${ }^{\star} p<0.05$.

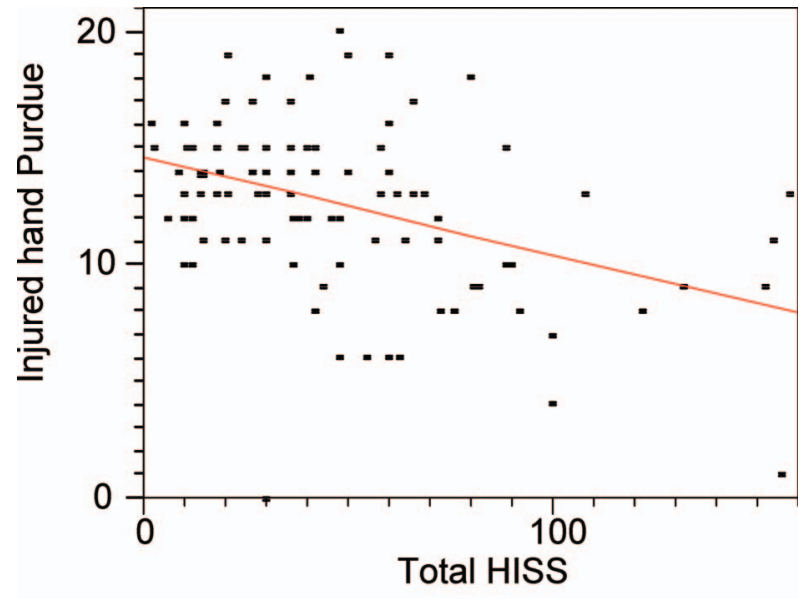

Injured hand Purdue $=14.62-0.04 \quad$ Total HISS $(p<0.01)$

Figure 1. Relationship between injured-hand Purdue after recovery and the total HISS score immediately after injury.

HISS score, but not the individual components. The skeletal component was associated with the injuredhand and both-hands subtests, but not the assembly subtest. The neural component was associated with the assembly subtest (Table V).

We further analyzed the correlation between the severity categories of HISS and poor hand function. The risk of having low score in the injured-hand subtest was significantly increased in workers with initial major severity. The risk of having low score in the both-hands subtest was significantly increased in workers with initial severe and major severity. The risk of having low score in the assembly subtest was significantly increased in workers with initial major severity (Table VI).

\section{Discussion and conclusion}

This is the first study using an anatomical evaluation system to predict hand function after occupational hand injury. We found that initial hand injury severity as measured by the HISS system could predict hand function after an adequate recovery period.

Although this study was retrospective, information bias has been cautiously prevented by blinding the chart-reviewer, i.e., the hand surgeon from the study subjects' hand function results. The chart records included photographs of the injury upon arrival at the emergency department and notes of the findings during operation. The operation records were required by the Universal Health Insurance Board to be as thoroughly as possible for reimbursement purpose. Therefore, information relevant to the HISS components was readily available from the chart.

The HISS system comprehensively evaluated the injury anatomy. The scores of the HISS system represented the initial anatomic severity. Although the total HISS score had a wide possible range $(0-$ 1064), those of our subjects located in only lowest fifth range. The distribution was similar to previous reports $[11-13]$. The most severity category was the moderate severity $(40.0 \%)$ and the least category was the major severity $(8.4 \%)$. The distribution was also reasonable although the percentage of the major severity was less than previous reports $[12,13,16]$.

This study used Purdue Pegboard as a measurement of hand function status after recovery. Scores of the injured-hand, both-hands, and assembly were obtained. These three subsets represented different levels of difficulty in tasks. While comparing the performance of our subjects to the norm from male 
Table V. Correlation between the HISS scores and the Purdue Pegboard scores after adjustment for covariates.

\begin{tabular}{|c|c|c|c|c|c|c|c|c|c|}
\hline \multirow{2}{*}{$\begin{array}{l}\text { HISS } \\
\text { component }\end{array}$} & \multicolumn{3}{|c|}{ Injured-hand ${ }^{\dagger}$} & \multicolumn{3}{|c|}{ Both-hands } & \multicolumn{3}{|c|}{ Assembly ${ }^{*}$} \\
\hline & $\beta$ & $\mathrm{SE}$ of $\beta$ & $p$ & $\beta$ & SE of $\beta$ & $p$ & $\beta$ & SE of $\beta$ & $p$ \\
\hline Total score & -0.04 & 0.01 & $<0.01^{\star}$ & -0.03 & 0.01 & $<0.01^{\star}$ & -0.08 & 0.02 & $<0.01^{\star}$ \\
\hline Integument $^{\S}$ & -0.04 & 0.02 & 0.06 & -0.03 & 0.02 & 0.07 & -0.02 & 0.06 & 0.80 \\
\hline Skeletal $^{\S}$ & -0.04 & 0.02 & $0.05^{\star}$ & -0.04 & 0.01 & $0.01^{\star}$ & -0.09 & 0.05 & 0.08 \\
\hline Motor $^{\S}$ & -0.05 & 0.03 & 0.07 & -0.00 & 0.02 & 0.86 & -0.03 & 0.07 & 0.67 \\
\hline Neural $^{\S}$ & -0.01 & 0.05 & 0.79 & -0.03 & 0.04 & 0.37 & -0.33 & 0.14 & $0.02^{\star}$ \\
\hline
\end{tabular}

${ }^{\star} p<0.05 ;{ }^{\dagger}$ Adjusted for sex, age, education, dominant hand injury or not, uninjured hand Purdue; ${ }^{\star}$ Adjusted for sex, age, education, dominant hand injury or not; ${ }^{\S}$ Adjusted for all other components among integument, skeletal, motor and neural.

Table VI. The risk of having low score in the Purdue Pegboard according to the HISS severity after adjustment for covariates (gender, age, education, dominant hand injury or not).

\begin{tabular}{|c|c|c|c|c|c|c|c|c|c|c|}
\hline \multirow{2}{*}{$\begin{array}{l}\text { HISS } \\
\text { severity (score) }\end{array}$} & \multirow[b]{2}{*}{$n(\%)$} & \multicolumn{3}{|c|}{ Injured-hand } & \multicolumn{3}{|c|}{ Both-hands } & \multicolumn{3}{|c|}{ Assembly } \\
\hline & & OR & $95 \% \mathrm{CI}$ of $\mathrm{OR}$ & $p$ & OR & $95 \% \mathrm{CI}$ of $\mathrm{OR}$ & $p$ & OR & $95 \%$ CI of OR & $p$ \\
\hline Minor $(\leq 20)$ & $23(24.2)$ & & Reference & & & Reference & & & Reference & \\
\hline Moderate $(21-50)$ & $38(40.0)$ & 1.41 & $0.42-5.03$ & 0.58 & 2.08 & $0.52-9.82$ & 0.32 & 1.39 & $0.35-6.21$ & 0.65 \\
\hline Severe $(51-100)$ & $26(27.4)$ & 3.23 & $0.92-12.41$ & 0.07 & 4.50 & $1.10-21.78$ & $0.04^{\star}$ & 2.66 & $0.61-12.72$ & 0.20 \\
\hline Major $(\geq 101)$ & $8(8.4)$ & 9.57 & $1.40-94.80$ & $0.03^{\star}$ & 25.00 & $3.53-262.77$ & $<0.01^{\star}$ & 8.99 & $1.30-72.46$ & $0.03^{\star}$ \\
\hline
\end{tabular}

${ }^{\star} p<0.05$; OR, odds ratio; CI, confidence interval.

hourly production workers, more than one-third of our subjects did not achieve the 5 percentile level of the norm. Those with such low scores are candidates for early intensive rehabilitation [38].

All 3 subsets in Purdue Pegboard had scores negatively associated with initial HISS score. It implied that after at least half year of recovery, patients with higher initial anatomic severity regained lower hand function scores. However, risk of having poor performance (poorer than 5 percentile of normal workers) in the 3 subsets appeared to associate with different HISS severities (Table VI). The injuredhand subset showed direct effect of hand injury on hand function. The risk of poor performance was noted in patients with initial major severity (HISS > 100), and borderline significant in patients with severe HISS $(51-100)$. In the assembly subset, the sound hand could compensate for the clumsiness of the injured hand, and the risk of poor performance was noted only in patients with HISS $>100$. The both-hands subtest required both hands to work simultaneously, and was thus more difficult to execute [35]. Poor performance was noted in patients with HISS of $>50$.

In our study, we found not all individual components of the HISS system correlated with the hand function. Similar result was also reported [16]. Saxena reported only skeletal and motor components, but not neural component, were associated with the DASH. We revealed the skeletal component was associated with the injured-hand and both-hands subtests, but not the assembly subtest. The neural component was found to be associated with the assembly subtest. The explanation may be more than half of our subjects were not injured (i.e., scored zero) in motor and neural components and hand function performance of these subjects would be affected by other components. So the association between these components and the hand function would be masked. The other explanation is that overall evaluation to predict the outcome is better than evaluate on only certain components. The total HISS score is thus more valuable than score of individual component.

A rehabilitation staff concerns very much the recovery of hand function. Hand is an important but complicated part of human body. Both skeleton and soft tissue (tendon, ligament, nerve, skin) play their important roles. Since an injury seldom involves a single component, one cannot evaluate the severity by using any single structure. Interactions among the hand components have been well known to affect the overall hand performance [6-10]. Since the HISS system provides comprehensive evaluation of the severity of injury, as well as prediction of hand function after recovery, it can be used as an early indicator for setting a reasonable final goal of rehabilitation. In addition, early identification of patients needing more intensive rehabilitation will potentially improve clinical outcomes among the most severely injured. 
In conclusion, initial anatomical severity after occupational hand injury, evaluated by the HISS system, may predict hand function as evaluated by the Purdue Pegboard. Subjects with the initial total HISS score greater than 50 may have high risk of having poor hand function after recovery. Intensive rehabilitation is suggested. Further investigation with more study subjects will be warranted to distinguish effects of the HISS components on hand function after recovery.

\section{Acknowledgements}

The authors would like to thank the Bureau of Labor Insurance of Taiwan for grant to make this study possible.

\section{References}

1. Liu YH, Lin MR, Wang JD. Cost and determinants of morbidity from work related disabling injuries in Taiwan. Occup Environ Med 1995;52:138-142.

2. Fayad F, Nuwayhid I, Tamim H, Kassak K, Khogali M. Cost of work-related injuries in insured workplaces in Lebanon. Bull World Health Organ 2003;81:509-516.

3. Yearly report. Taiwan, ROC: Council of Labor Affairs, Executive Yuan; 2004.

4. O'Sullivan ME, Colville J. The economic impact of hand injuries. J Hand Surg [Br] 1993;18B:395-398.

5. Schutt AH, Bengtson KA. Hand rehabilitation. In: DeLisa JA, Gans BM, editors. Rehabilitation medicine: Principles and practice. Philadelphia: Lippincott-Raven Publishers; 1998. pp 1717-1719.

6. Huffaker WH, Wray RC, Weeks PM. Factors influencing final range of motion in the fingers after fractures of the hand. Plast Reconstr Surg 1979;63:82-87.

7. Chow SP, Pun WK, So YC, Luk KDK, Chid KY, Ng KH, et al. A prospective study of 245 open digital fractures of the hand. J Hand Surg [Br] 1991;16B:137-140.

8. van Oosterom FJT, Ettema AM, Mulder PGH, Hovius SER. Functional outcome after surgical treatment of phalangeal fractures in severely injured hands. Scand J Plast Reconstr Surg Hand Surg 2005;39:238-241.

9. Chin G, Weinzweig N, Mead M, Gonzalez M. "Spaghetti wrist": Management and results. Plast Reconstr Surg 1998; 102:96-102.

10. Jaquet J-B, Luijsterburg AJM, Kalmijn S, Kuypers PDL, Hofman A, Hovius SER. Median, ulnar, and combined median-ulnar nerve injuries: Functional outcome and return to productivity. J Trauma 2001;51:687-692.

11. Campbell DA, Kay SPJ. The hand injury severity scoring system. J Hand Surg [Br] 1996;21B:295-298.

12. Mink van der Molen AB, Matloub HS, Dzwierzynski W, Sanger JR. The hand injury severity scoring system and workers' compensation cases in Wisconsin, USA. J Hand Surg [Br] 1999;24B:184- 186.

13. Mink van der Molen AB, Ettema AM, Hovius SER. Outcome of hand trauma: The hand injury severity scoring system (HISS) and subsequent impairment and disability. J Hand Surg [Br] 2003;28B:295-299.

14. Hudak PL, Amadio PC, Bombardier C, Beaton D, Cole D, Davis A, et al. Development of an upper extremity outcome measure: The DASH (disabilities of the arm, shoulder, and head). Am J Ind Med 1996;29:602-608.
15. Adam SD, Belinda JG, Owen DW, Peter AC. Outcome instruments for the assessment of the upper extremity following trauma: A review. Injury 2005;36:468-476.

16. Saxena P, Cutler L, Feldber L. Assessment of the severity of hand injuries using 'hand injury severity score', and its correlation with the functional outcome. Int J Care Injured 2004;35:511-516.

17. World Health Organization. International Classification of Functioning, Disability and Health. ICF. ed. Geneva: World Health Organization; 2001.

18. Kärki A, Simonen R, Mälkiä E, Selfe J. Impairments, activity limitations and participation restrictions 6 and 12 months after breast cancer operation. J Rehabil Med 2005;37:180 - 188.

19. Eriksson G, Kerstin T, Borg J. Occupational gaps in everyday life $1-4$ years after acquired brain injury. J Rehabil Med 2006; 38:159-165.

20. Trombly CA, Scott AD. Evaluation of motor control. In: Trombly CA, editor. Occupational therapy for physical dysfunction. Baltimore: Williams \& Wilkins; 1989. p 68.

21. Tiffin J, Asher EJ. The Purdue pegboard: Norms and studies of reliability and validity. J Appl Psychol 1948;32:234-247.

22. Hines M, O'Connor J. A measure of finger dexterity. Personnel J 1962;4:379-382.

23. Jebsen RH, Taylor N, Trieschmann RB, Trotter MJ, Howard LA. An objective and standardized test of hand function. Arch Phys Med Rehabil 1969;50:311-319.

24. Minnesota Rate of Manipulation Test examiner's manual. Circle Pines, MN: American Guidance Service, Inc; 1969.

25. Smith HB. Smith Hand Function Evaluation. Am J Occup Ther 1973;27:244-251.

26. Bennett GK. Hand tool dexterity test manual. New York, Harcourt Brace Jovanovich; 1981.

27. Mathiowetz V, Weber K, Kashman N, Volland G. Adult norms for Nine Hole Peg Test of Finger Dexterity. Occup Ther J Res 1985;5:25-38.

28. Desrosiers J, Hebert R, Dutil E. TEMPA Administration Manual. Sherbrook, Quebec, Centre de reserche interdisciplinaire de l'Hôpital d'Youvillé affilié al "Universitié de Sherbrooke; 1991.

29. Tiffin J. Purdue Pegboard Examiner Manual. Chicago, IL: Science Research Associates; 1968.

30. Sawyer J, Bennett A. Comparing the level of dexterity offered by latex and nitrile SafeSkin gloves. Ann Occup Hyg 2005: $1-8$.

31. Irvine J, Chong SL, Amirjani N, Chan KM. Double-blind randomized controlled trial of low-level laser therapy in carpal tunnel syndrome. Muscle Nerve 2004;30:182 - 187.

32. Pastores GM, Barnett NL, Kolodny EH. An open-label, noncomparative study of miglustat in type I Gaucher disease: Efficacy and tolerability over 24 months of treatment. Clin Ther 2005;27:1215-1227.

33. Worringham CJ, Wood JM, Kerr GK, Silburn PA. Predictors of driving assessment outcome in Parkinson's disease. Mov Disord 2006;21:230-235.

34. Reddon JR, Gill DM, Gauk SE, Maerz MD. Purdue pegboard: Test-retest estimates. Percept Mot Skills 1988;66: 503-506.

35. Desrosiers J, Hébert R, Bravo G, Dutil E. The Purdue Pegboard Test: Normative data for people aged 60 and over. Disabil Rehabil 1995;17:217-224.

36. Buddenberg LA, Davis C. Test-retest reliability of the Purdue Pegboard test. Am J Occup Ther 2000;54:555-558.

37. Instructions and normative data for MODEL 32020 PURDUE PEGBOARD. Lafayette, IN: Lafayette instrument company.

38. Sampaio RF, Mancini MC, Silva FCM, Figueiredo IM, Vaz DV, Alves GBDO. Work-related hand injuries: Case analyses in a Brazilian rehabilitation service. Disabil Rehabil 2006;28: $803-808$. 